MOUSE MODELS

\section{One step closer...}

Ataxia telangiectasia - caused by homozygous mutations in ATMhas long been linked with radiosensitivity, genomic instability and a predisposition to cancer, but do heterozygous individuals also share these characteristics? Martin Lavin and colleagues, reporting in the September issue of Nature Genetics, have used a mouse model to investigate this question, and have moved one step closer to the answer.

An ATM missense mutation 7636del9, which results in the inframe deletion of three amino acids — has been identified in individuals with ataxia telangiectasia. The corresponding homozygous mutation in mice (Atm-SSRI) also increases tumour incidence. So what about mice that are heterozygous for Atm- $\Delta$ SRI? These also showed an increase in tumour formation $-8.9 \%$ compared with $2.8 \%$ in wild-type littermates - and the tumour spectrum was similar, but not identical, to that found in mice that were homozygous for this mutation. Humans who were heterozygous for this mutation were analysed for tumour incidence, and were shown to exhibit a small increase; however, this was not statistically significant. Might these individuals, instead, display some of the other features of ataxia telangiectasia?

Heterozygous human and mouse cells were isolated, and were examined for survival and genomic instability following exposure to radiation. The level of radiation-induced death and the number of chromosome aberrations were found to be intermediate between wild-type and homozygous-mutant cells.

The ATM protein kinase protects cells from DNA damage by phosphorylating and activating proteins such as $\mathrm{p} 53$. So is this ability impaired in $A T M-\triangle$ SRI mutants? Wild-type or $A T M-\Delta$ SRI DNA was transfected into control lymphoblastoid cells, and kinase assays were performed on immunoprecipitated ATM, using p53 as a substrate. Kinase activity was abolished in cells that were transfected with the $\Delta$ SRI mutant, indicating that it acts as a dominant-negative inhibitor of the wild-type protein. This lends support to the finding that mice that are heterozygous for an Atm deletion do not have an increased tumour incidence, and indicates that cancer predisposition in heterozygous carriers of $A T M$ mutants could crucially depend on the specific mutation involved.

Hopefully, the cancer risk of other carriers of ATM mutations will soon be accurately determined, so that individuals are able to take any recommended precautions. Emma Greenwood

(2) References and links ORIGINAL RESEARCH PAPER Spring, K. et al. Mice heterozygous for mutation in Atm, the gene involved in ataxia-telengiectasia, have heightened susceptibility to cancer. Nature Genet. 32, 185-190 (2002)

FURTHER READING Concannon, P. ATM heterozygosity and cancer risk. Nature Genet. 32 89-90 (2002)

WEB SITE

Martin Lavin's's lab:

http://www.qimr.edu.au/research/labs/martin//

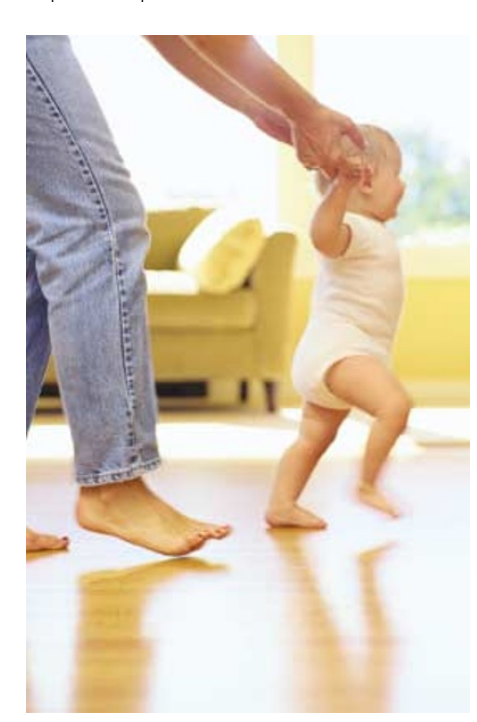

\section{TRIAL WATCH}

\section{SAHA sails ahead}

Encouraging results have been reported from Phase I clinical trials of suberoylanilide hydroxamic acid (SAHA) - an inhibitor of histone deacetylase (HDAC). The drug was given orally or intravenously to 70 patients with solid and haematological tumours and shown to have excellent oral bioavailability, long duration of action and to inhibit HDAC at doses that were well tolerated. The studies, which were conducted at the Memorial Sloan-Kettering Cancer Center, were reported by Victoria Richon of Aton Pharma at the First International Congress on Targeted Therapies in Washington DC (16-18 August, 2002).

HDACs catalyse the removal of acetyl groups from core nucleosomal histones to alter chromatin structure and regulate the transcriptional activity. Hypoacetylated histones are generally associated with transcriptional repression, and aberrant recruitment of HDAC activity has been associated with the development of certain human cancers. SAHA inhibits HDAC activity by directly binding the catalytic pocket of the enzyme, leading to the accumulation of acetylated core nucleosomal histones.

The investigators are planning Phase II studies of SAHA, and a second HDAC inhibitor, pyroxamide, is already in Phase I trials that are sponsored by the National Cancer Institute.

\section{Wait or act?}

Radical prostatectomy is widely used for the treatment of early prostate cancer, but it has not been adequately assessed in a randomized trial until now. Holmberg et al., of the Scandinavian Prostatic Cancer Group, report that the outcomes of radical prostatectomy and watchful waiting are similar.

A total of 695 men with early prostate cancer were randomized to watchful waiting or radical prostatectomy. After a median followup of 6.2 years, there was no significant difference in overall survival between the two groups. However, death due to prostate cancer was higher in the group that was assigned to watchful waiting - the relative risk of death due to prostate cancer after 8 years of followup was 0.5 . The authors conclude that the absolute benefit associated with radical surgery is limited in men who have the same risk of dying from prostate cancer as the men in this study.

An accompanying article by Steineck $e t$ al. indicates that the effects on well-being and subjective quality of life in the men in the trial discussed above, evaluated 4 years after randomization, were similar in both groups.

This randomized trial was initiated before screening for prostatespecific antigen began, and Holmberg et al. suggest that, in men with cancer detected by screening — which would be more representative of most patients today — the base-line risk of death from prostate cancer might be even lower, and the benefit of radical treatment might therefore also be less. Longer follow-up is awaited, as metastases and death in patients with prostate cancer can occur up to 20 or 25 years after diagnosis. In addition, in the past decade, radiation-therapy techniques have been developed as an alternative treatment approach, and are soon to be evaluated in randomized trials.

ORIGINAL RESEARCH PAPERS Holmberg, L et al. A randomized trial comparing radical prostatectomy with watchful waiting in early prostate cancer. N. Engl. J. Med. 347, 781-789 (2002) | Steinbeck, G. et al. Quality of life after radical prostatectomy or watchful waiting. N. Engl. J. Med. 347, 790-796 (2002) 